\title{
Improved Soundings and Error Estimates using AIRS/AMSU Data
}

\author{
Joel Susskind \\ NASA Goddard Space Flight Center, Greenbelt, MD, USA 20771
}

\section{Popular Summary}

AIRS was launched on EOS Aqua on May 4, 2002, together with AMSU A and HSB, to form a next generation polar orbiting infrared and microwave atmospheric sounding system. The primary products of AIRS/AMSU are twice daily global fields of atmospheric temperature-humidity profiles, ozone profiles, sea/land surface skin temperature, and cloud related parameters. Also included are the clear column radiances used to derive these products which are representative of the radiances AIRS would have seen if there were no clouds in the field of view. All products also have error estimates. The sounding goals of AIRS are to produce $1 \mathrm{~km}$ tropospheric layer mean temperatures with an rms error of $1 \mathrm{~K}$, and layer precipitable water with an rms error of 20 percent, in cases with up to 80 percent effective cloud cover. The products are designed for data assimilation purposes for the improvement of numerical weather prediction, as well as for the study of climate and meteorological processes. With regard to data assimilation, one can use either the products themselves or the clear column radiances from which the products were derived.

A post-launch algorithm, referred to as AIRS Version 4.0, has been used by the Goddard DAAC to analyze and distribute AIRS retrieval products. In this paper, progress is shown toward the AIRS Version 5.0 algorithm which will be used by the Goddard DAAC to analyze past and future AIRS/AMSU data starting late in 2006. A new methodology has been developed to provide accurate case by case error estimates for retrieved geophysical parameters and for the channel by channel cloud cleared radiances used to derive the geophysical parameters from the AIRS/AMSU observations. An accurate knowledge of individual error estimates enhances the value of the retrieved products, both from the data assimilation and climate assessment perspectives. .These error estimates are in turn used for quality control of the derived geophysical parameters and clear column radiances. Version 5.0 retrieval accuracy and spatial coverage of quality controlled retrieval products are shown to be superior to those obtained using Version 4.0. 


\title{
Improved Soundings and Error Estimates using AIRS/AMSU Data
}

\author{
Joel Susskind \\ NASA Goddard Space Flight Center, Greenbelt, MD, USA 20771
}

\begin{abstract}
AIRS was launched on EOS Aqua on May 4, 2002, together with AMSU A and HSB, to form a next generation polar orbiting infrared and microwave atmospheric sounding system. The primary products of AIRS/AMSU are twice daily global fields of atmospheric temperature-humidity profiles, ozone profiles, sea/land surface skin temperature, and cloud related parameters including OLR. The sounding goals of AIRS are to produce $1 \mathrm{~km}$ tropospheric layer mean temperatures with an $\mathrm{rms}$ error of $1 \mathrm{~K}$, and layer precipitable water with an rms error of 20 percent, in cases with up to 80 percent effective cloud cover. The basic theory used to analyze AIRS/AMSU/HSB data in the presence of clouds, called the at-launch algorithm, and a post-launch algorithm which differed only in the minor details from the at-launch algorithm, have been described previously. The post-launch algorithm, referred to as AIRS Version 4.0, has been used by the Goddard DAAC to analyze and distribute AIRS retrieval products. In this paper we show progress made toward the AIRS Version 5.0 algorithm which will be used by the Goddard DAAC starting late in 2006. A new methodology has been developed to provide accurate case by case error estimates for retrieved geophysical parameters and for the channel by channel cloud cleared radiances used to derive the geophysical parameters from the AIRS/AMSU observations. These error estimates are in turn used for quality control of the derived geophysical parameters and clear column radiances. Improvements made to the retrieval algorithm since Version 4.0 are described as well as results comparing Version 5.0 retrieval accuracy and spatial coverage with those obtained using Version 4.0.
\end{abstract}

Keywords: infra-red, microwave, remote sensing, temperature, moisture, high spectral resolution

\section{INTRODUCTION}

AIRS was launched on EOS Aqua on May 4, 2002, together with AMSU A and HSB, to form a next generation polar orbiting infrared and microwave atmospheric sounding system. ${ }^{1}$ The primary products of AIRS/AMSU are twice daily global fields of atmospheric temperature-humidity profiles, ozone profiles, sea/land surface skin temperature, and cloud related parameters including OLR. Also included are the clear column radiances used to derive these products which are representative of the radiances AIRS would have seen if there were no clouds in the field of view. All products also have error estimates. The sounding goals of AIRS are to produce $1 \mathrm{~km}$ tropospheric layer mean temperatures with an rms error of $1 \mathrm{~K}$, and layer precipitable water with an rms error of 20 percent, in cases with up to 80 percent effective cloud cover. The products are designed for data assimilation purposes for the improvement of numerical weather prediction, as well as for the study of climate and meteorological processes. With regard to data assimilation, one can use either the products themselves or the clear column radiances from which the products were derived.

The basic theory used to analyze AIRS/AMSU/HSB data in the presence of clouds, called the at-launch algorithm, and that used in a post-launch algorithm which differed only in the minor details from the at-launch algorithm, have been described previously ${ }^{2,3}$. The post-launch algorithm, referred to as AIRS Version 4.0, has been used by the Goddard DAAC to analyze and distribute AIRS retrieval products. In this paper we show progress made toward the AIRS Version 5.0 algorithm which will be used by the Goddard DAAC starting late in 2006. A new methodology has been developed to provide accurate case by case error estimates for retrieved geophysical parameters and for the channel by channel cloud cleared radiances used to derive the geophysical parameters from the AIRS/AMSU observations. These error estimates are in turn used for quality control of the derived geophysical parameters and clear column radiances. Improvements made to the retrieval algorithm since Version 4.0 are described as well as results comparing Version 5.0 retrieval accuracy and spatial coverage with those obtained using Version 4.0. 


\section{OVERVIEW OF THE AIRS TEAM RETRIEVAL ALGORITHM}

The AIRS team candidate Version 5.0 retrieval algorithm is basically identical to that described in Susskind et al. ${ }^{2,3}$. The key steps are outlined below: 1) Start with an initial state, called the MW retrieval state, consistent with the AMSU A and HSB radiances ${ }^{4}$; 2) Derive IR clear column radiances $\hat{R}_{i}^{0}$ valid for the $3 \times 3$ AIRS Fields of View (FOVs) within an AMSU A Field of Regard (FOR) consistent with the observed radiances and the initial state; 3) Obtain an AIRS regression guess ${ }^{5}$ consistent with $\hat{R}_{i}^{0}$ using 1504 AIRS channels; 4) Derive $\hat{R}_{i}^{1}$ consistent with the AIRS radiances and the regression guess; 5) Derive all surface and atmospheric parameters using $\hat{R}_{\mathrm{i}}^{1}$ for 293 AIRS channels and all AMSU radiances; 6) Derive an improved set of clear column radiances $\hat{R}_{i}^{2}$ using the AIRS physically retrieved parameters; 7) Repeat Step 5 using $\hat{R}_{i}^{2}$ to produce the final IR/MW retrieval state; 8) Derive cloud parameters and OLR consistent with the solution and observed $R_{i}$; 9) Apply initial quality control, which rejects a IR/MW solution if the retrieved cloud fraction is greater than $90 \%$ or other relatively coarse tests fail. In the event that a retrieval is rejected initially, cloud parameters are determined consistent with the initial MW retrieved state and observed AIRS radiances. Otherwise, cloud parameters are computed based on the coupled IR/MW retrieval, and further quality control is applied to individual geophysical parameters.

Candidate Version 5.0 differs from Version 4.0 with regard to some details in the physical retrieval steps. The major difference between candidate Version 5.0 and Version 4.0 is new methodology to determine accurate case by case, parameter by parameter, error estimates and, in addition, the use of these for quality control. The next two sections describe some of the changes in the details of the retrieval algorithm and give the methodology for generation of error estimates and their use in quality control.

\section{MODIFICATIONS TO THE VERSION 4.0 RETRIEVAL ALGORITHM}

The most significant improvement to the AIRS Science Team Version 4.0 retrieval algorithm is the use of a new AIRS Radiative Transfer Algorithm (RTA) which contains improved characterization of atmospheric absorption characteristics, and more significantly, allows for effects of non Local Thermodynamic Equilibrium (non-LTE) which affects radiances in most shortwave temperature sounding channels during the day. Two important consequences of these improvements are that the needed systematic radiative transfer bias error correction coefficients (referred to as tuning coefficients) are now greatly reduced from those of Version 4.0, and in addition, many shortwave temperature sounding channels sensitive to non-LTE can now be (and are)
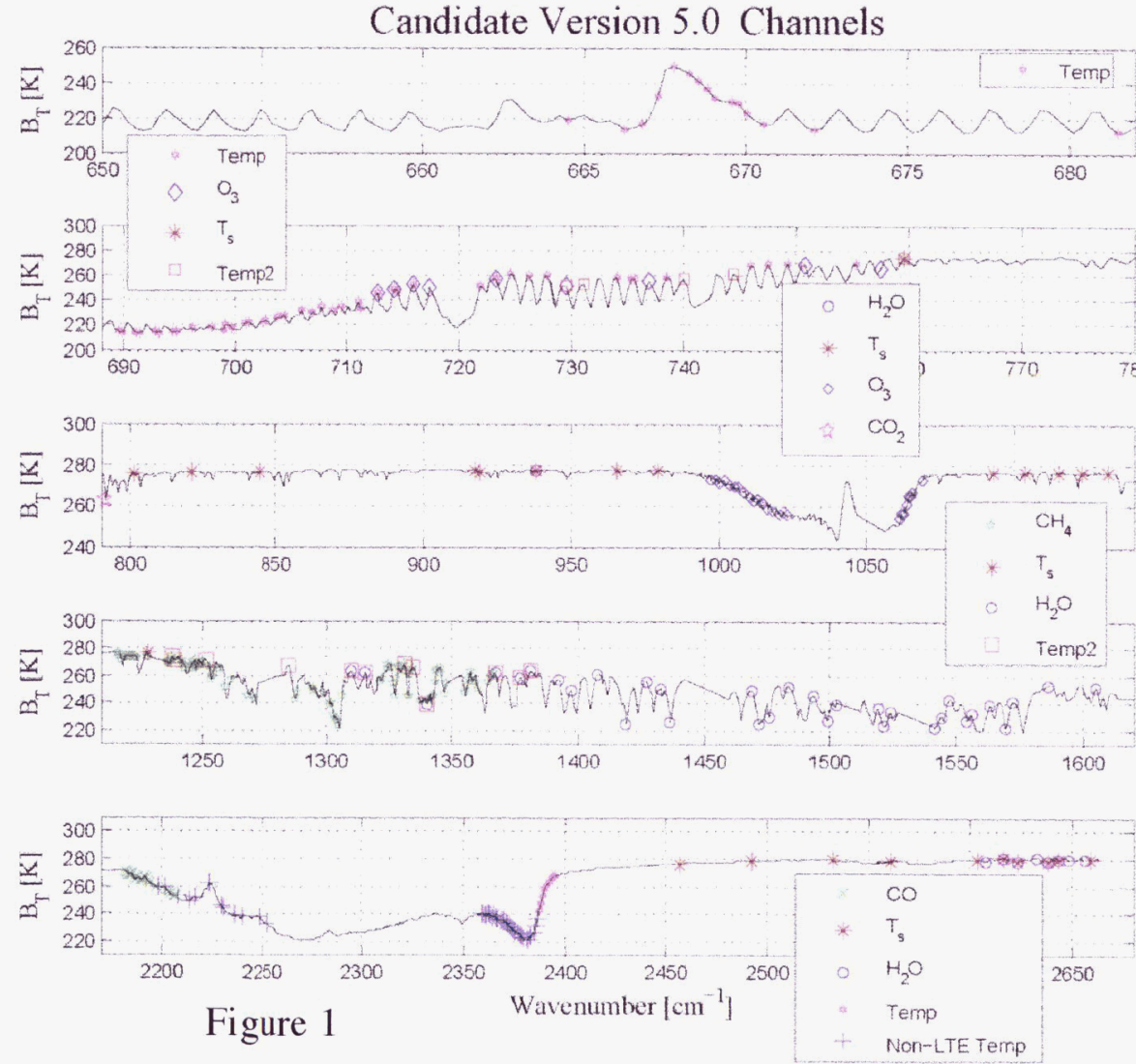
used effectively, both day and night, in the physical retrieval process.

Figure 1 shows a typical AIRS brightness temperature spectrum and includes the channels used in Version 5.0 in different steps of the AIRS physical retrieval algorithm. The location and number of channels used are somewhat different from those of Version 4.0. The major difference is in the incorporation of the non-LTE temperature sounding channels in the temperature profile retrieval system, as indicated in Figure 1.

The basic cloud clearing methodology used to determine $\hat{\mathrm{R}}_{\mathrm{i}}$ is unchanged from that described in Susskind et al. ${ }^{2,3}$. In this methodology, a set of cloud clearing channels is used, in conjunction with an nth estimate of the geophysical state, to

generate $\hat{R}_{i}^{n}$. The cloud clearing channels used are sensitive to atmospheric absorption and emission at all levels of the troposphere, including emission from the earth's surface. Inclusion of channels very sensitive to emission from the earth's surface is useful to account for low clouds in the field of view, and a number of such (window) channels were used for cloud clearing over both land and ocean in Version 4.0. It has been subsequently determined that while surface channels improve cloud clearing ability over ocean, they have detrimental effects over land resulting from variable subpixel land skin temperatures and surface emissivities. Consequently, in candidate Version 5.0, cloud clearing channels with high sensitivity to the surface are not used over land (but are still used over ocean).

\section{ERROR ESTIMATES AND QUALITY CONTROL}

Coupled AIRS/AMSU retrievals in the presence of broken cloud cover are usually highly accurate. Under some conditions, such as complete overcast, combined AIRS/AMSU retrievals cannot be performed at all. In cases of complex clouds or terrain, retrievals are of poorer quality. In the pre-launch version of the AIRS/AMSU retrieval algorithm, quality control was applied uniformly to the entire profile. If any geophysical parameter was considered to be of poor quality, the whole set of retrieval geophysical parameters was rejected and clouds were derived using the MW state of Step (1) above. This "one size fits all" approach led to significant compromises between desired spatial coverage of accepted retrievals and desired accuracy. In Version $4.0^{3}$, the combined IR/MW retrieval parameters is retained, and used to derive cloud parameters, as long as it is felt that the combined IR/MW retrieval (Step 7) is at least as accurate as the MW only retrieval (Step 1). This was considered to be true if the retrieved cloud fraction derived using the $\mathrm{M} / \mathrm{MW}$ state was less than or equal to $90 \%$ and the initial cloud clearing step was stable. If this test was passed (referred to as the Stratospheric Temperature Test), the temperature profile above $200 \mathrm{mb}$ was considered acceptable. Constituent profiles $\left(\mathrm{H}_{2} \mathrm{O}, \mathrm{O}_{3}, \mathrm{CO}\right.$, and $\left.\mathrm{CH}_{4}\right)$ were accepted if the Stratospheric Temperature Test was passed and additional slightly more stringent cloud clearing stability tests were also passed. The next level of test was applied to the temperature profile beneath $200 \mathrm{mb}$ and above $3 \mathrm{~km}$ (the Mid Tropospheric Temperature Test). Finally, a more stringent test was applied to accept temperature profiles in the lowest $3 \mathrm{~km}$ of the atmosphere (Lower Tropospheric Temperature Test). Lower tropospheric temperatures are the most difficult to determine accurately, both because of effects of low clouds on the radiances and uncertainty and small scale variability in surface skin temperature and emissivity. Both concerns create greater problems over land than ocean. In response to this, the Lower Tropospheric Temperature Test rejected lower tropospheric temperature more often over land than over ocean in Version 4.0.

\subsection{Error estimates and quality control for retrieved temperature profiles and surface skin temperatures}

The Version 4.0 quality control tests each used thresholds for values of 12 different parameters $Y_{k}(k=1,12)$ representative of residuals of internal convergence tests. In candidate Version 5.0, the case by case values of each of the parameters whose thresholds were used in the Version 4.0 acceptance tests, $Y_{k}$, are used in the generation of error estimates of the individual retrieved parameters. In the case of either $T(p)$ or $T_{\text {skin }}$, we write

$$
\delta \mathrm{T}_{\mathrm{i}}=\sum_{\mathrm{k}} \mathrm{M}_{\mathrm{ik}} \mathrm{Y}_{\mathrm{k}}
$$


where $\delta T_{i}$ is the temperature error estimate, $Y_{k}$ is the value of the $k^{\text {th }}$ test, and $M$ is a matrix with different values over ocean and land. Error estimates are, by definition, all positive.

If one knows the actual errors, given by $T_{i}-T_{i}^{\text {truth }}$, the matrix $M$ can be determined in a straightforward manner, by finding $M$ such that M minimizes the RMS difference of $\left(\Delta T_{i}-\delta T_{i}\right)$, where $\Delta T_{i}=\left|T_{i}-T_{i}^{\text {truth }}\right|$. In order to generate $M$, we used $T_{i}$ and $Y_{k}$ for all candidate Version 5.0 IR/MW retrievals (that is all cases passing the Version 4.0 Stratospheric Temperature Test) on September 29. 2004, and used the colocated ECMWF 3 hour forecast used as truth. The accuracy of the error estimates, and of their use for quality control, was tested on global candidate Version 5.0 retrievals run on January 25, 2003

\subsubsection{Surface skin temperature quality control}

Ocean surface skin temperatures are measured very accurately from other EOS instruments such as MODIS and AMSRE. AIRS ocean skin temperatures must be very accurate in order to provide additional useful information regarding sea surface temperature anomalies. In Version 4.0, sea surface temperatures were classified according to their ability to pass either a tight SST test (highest quality) or a standard SST test (good quality). Monthly mean products were generated by including all cases passing the standard SST test. In Version 5.0, we use the ocean skin temperature error estimate directly for quality control. We currently classify ocean skin temperatures as highest quality if the $\delta \mathrm{T}_{\text {skin }}<1$.OK and good quality if $\delta \mathrm{T}_{\text {skin }}<1.25 \mathrm{~K}$. Figure $2 \mathrm{a}$ shows the spatial distribution of non-frozen ocean surface skin temperature errors for ascending (daytime) orbits on January 25, 2003 for all cases in which the combined IR/MW retrievals were produced. The predicted errors, obtained from Equation 1, are shown in Figure 2b. Figure 2d shows the difference between the predicted error and the absolute value of the actual error. The largest errors are somewhat under-predicted and the smallest errors over-predicted, but the spatial correlation $(0.71)$ is very good. Figure $2 \mathrm{c}$ shows the spatial distribution of the errors for those sea surface temperatures classified as good $\left(\delta \mathrm{T}_{\text {skin }}<1.25 \mathrm{~K}\right.$ ). The current plan is to include these cases in the generation of the monthly mean SST product.

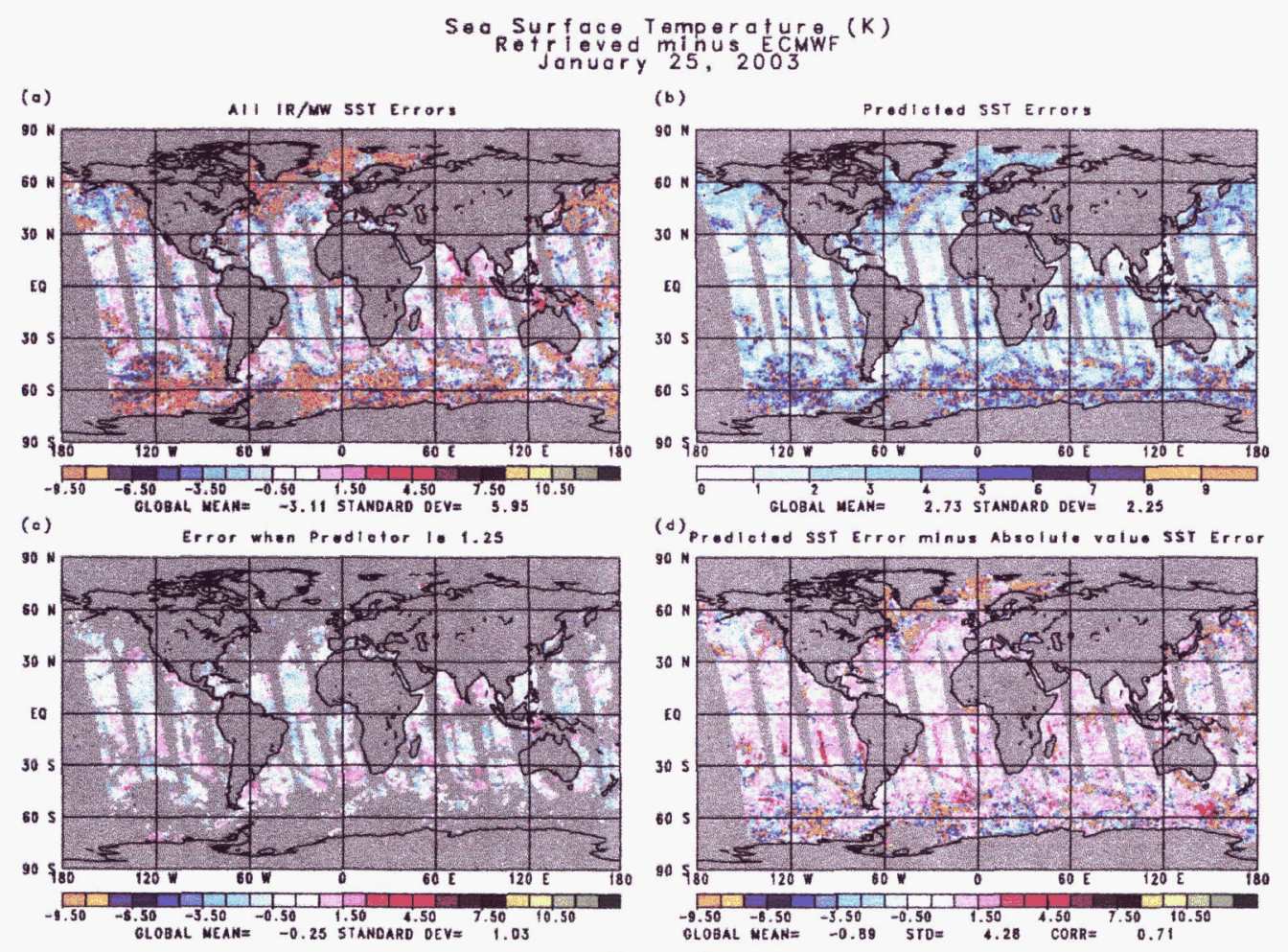

Figure 2 


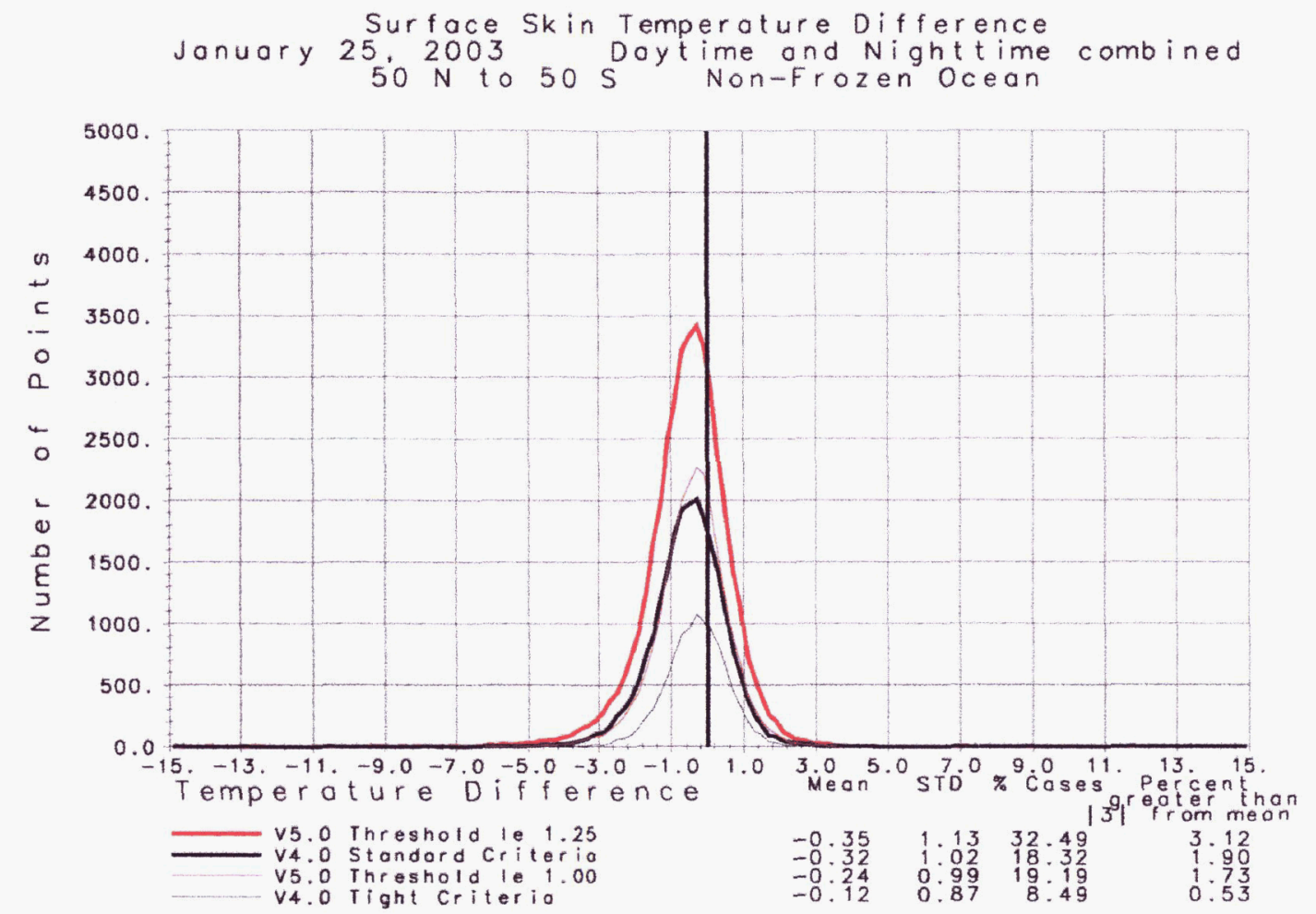

Figure 3

Figure 3 shows histograms of the distribution of quality controlled (both good and highest quality) ocean retrievals as a function of SST differences from ECMWF "truth" on January 25, 2003, obtained using Version 4.0 and candidate Version 5.0. The mean difference from ECMWF, the spatial standard deviation of the difference, the percent of all cases accepted, and the percent outliers (errors more than $3 \mathrm{~K}$ from the mean) are indicated in the figure. Candidate Version 5.0 has a higher yield than Version 4.0 for both the highest quality and the good quality retrievals.

Equation 1 is also used to generate $\delta \mathrm{T}_{\text {skin }}$ over land and ice. A caveat here is that the values of the ECMWF $\mathrm{T}_{\text {skin }}$ "truth" are considerably less accurate over land and ice than over ocean. Nevertheless, the procedure produces a reasonable spatial distribution of surface skin temperature error estimates over land and ice cases (not shown). These error estimates are not currently used for quality control of the land surface skin temperature, but are taken into account with regard to temperature profile quality control over land.

\subsubsection{Temperature profile quality control}

As with surface skin temperature, case by case level by level error estimate for temperature profile are also obtained by equation 1. We use these error estimates to determine a case by case characteristic pressure $\mathrm{p}_{\text {good }}$, down to which the profile is considered acceptable. We assume all IR/MW derived profiles are acceptable down to at least $70 \mathrm{mb}$. If the $\delta \mathrm{T}_{\text {skin }}$ is less than $2 \mathrm{~K}$ over either land or ocean, it is assumed that the cloud clearing is adequate to produce an accurate temperature profile and $p_{\text {good }}$ is set to be the surface pressure $p_{\text {surf }}$. Otherwise, the characteristic pressure $p_{\text {good }}$ is defined as the highest pressure (somewhere between $70 \mathrm{mb}$ and $\mathrm{p}_{\text {surf }}$ ) at which the error estimate at the next 3 pressure levels is not greater than a pressure dependent error estimate threshold. At the time of this paper, the error estimate 
thresholds are taken as $2.0 \mathrm{~K}$ at $70 \mathrm{mb}, 1.0 \mathrm{~K}$ at $\mathrm{p}_{\text {surf }} / 2$, and $1.0 \mathrm{~K}$ at $\mathrm{p}_{\text {surf }}$ over ocean, and $2 \mathrm{~K}$ at $70 \mathrm{mb}, 1.25 \mathrm{~K}$ as $\mathrm{p}_{\text {surf }} / 2$, and $1.5 \mathrm{~K}$ at $\mathrm{p}_{\text {surf }}$ over land, with values linearly interpolated in $\ell \mathrm{np}$ at intermediate values.

Figure 4a shows the differences of retrieved $300 \mathrm{mb}$ temperatures from ECMWF "truth" for all ascending orbit retrieval IR/MW cases in January 25, 2003. Gray means missing data. This can be a result of orbit gaps, a missing granule (over central Africa), or (generally very cloudy) areas where successful IR/MW retrievals were not performed (such as off the northwest coast of the U.S.). The area weighted global mean of the error is $-.01 \mathrm{~K}$, and its spatial standard deviation is $1.54 \mathrm{~K}$. Figure $4 \mathrm{~b}$ shows the predicted errors, and Figure $4 \mathrm{~d}$ shows the differences between the predicted error and the absolute value of the actual error. The spatial correlation is 0.53 , and the spatial standard deviation is 0.88 , showing reasonable skill between the actual "error" (which may itself be incorrect due to errors in the truth), and the predicted error. Figure $4 \mathrm{c}$ shows the $300 \mathrm{mb}$ error the quality controlled cases, i.e., cases in which $\mathrm{p}_{\mathrm{good}} \geq 300 \mathrm{mb}$. The spatial distribution of accepted cases is quite comprehensive, and the standard deviation of the errors for accepted cases has dropped to $1.11 \mathrm{~K}$. The largest "errors" for the accepted cases occur over Antarctica, Greenland, and Northern Siberia, in locations where (by definition), the error estimates are low. These are regions in which the ECMWF "truth" may be of poorer quality and actual errors may be less than the errors shown in Figure $4 \mathrm{c}$.

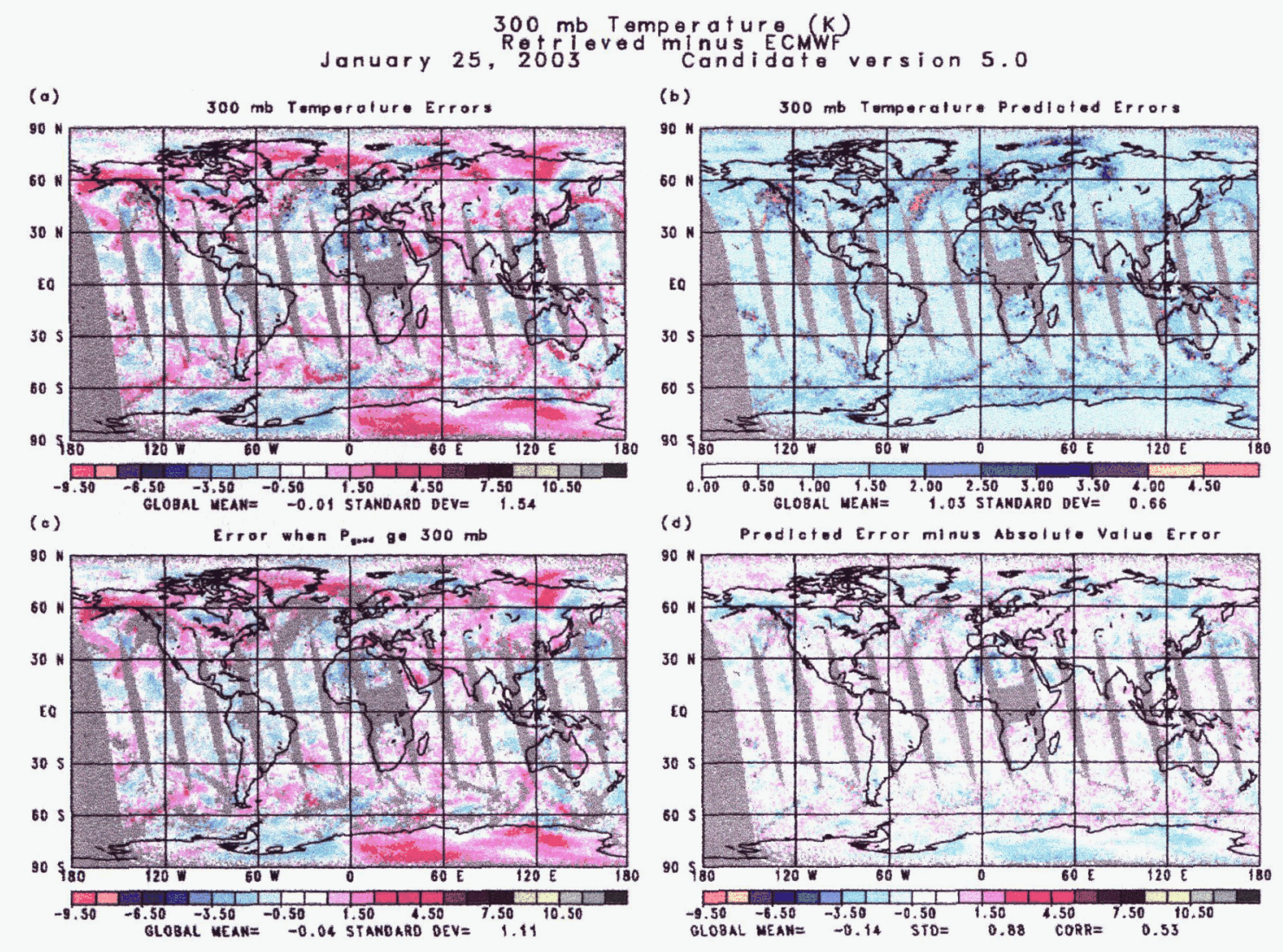

Figure 4

Figure 5 shows the spatial distribution of quality controlled retrieved $700 \mathrm{mb}$ temperatures and their errors vs. ECMWF "truth" for Version 4.0 and candidate Version 5.0 retrievals. Areas in which the surface pressure is less than $700 \mathrm{mb}$ are not included in the figure. The global standard deviation of quality controlled errors is somewhat larger in candidate Version 5.0 than in Version 4.0. It is important, however, to note that the spatial coverage over land and at high latitudes is significantly higher in candidate Version 5.0 than in Version 4.0, with reasonable $700 \mathrm{mb}$ temperature accuracies in these regions. 


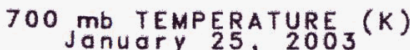
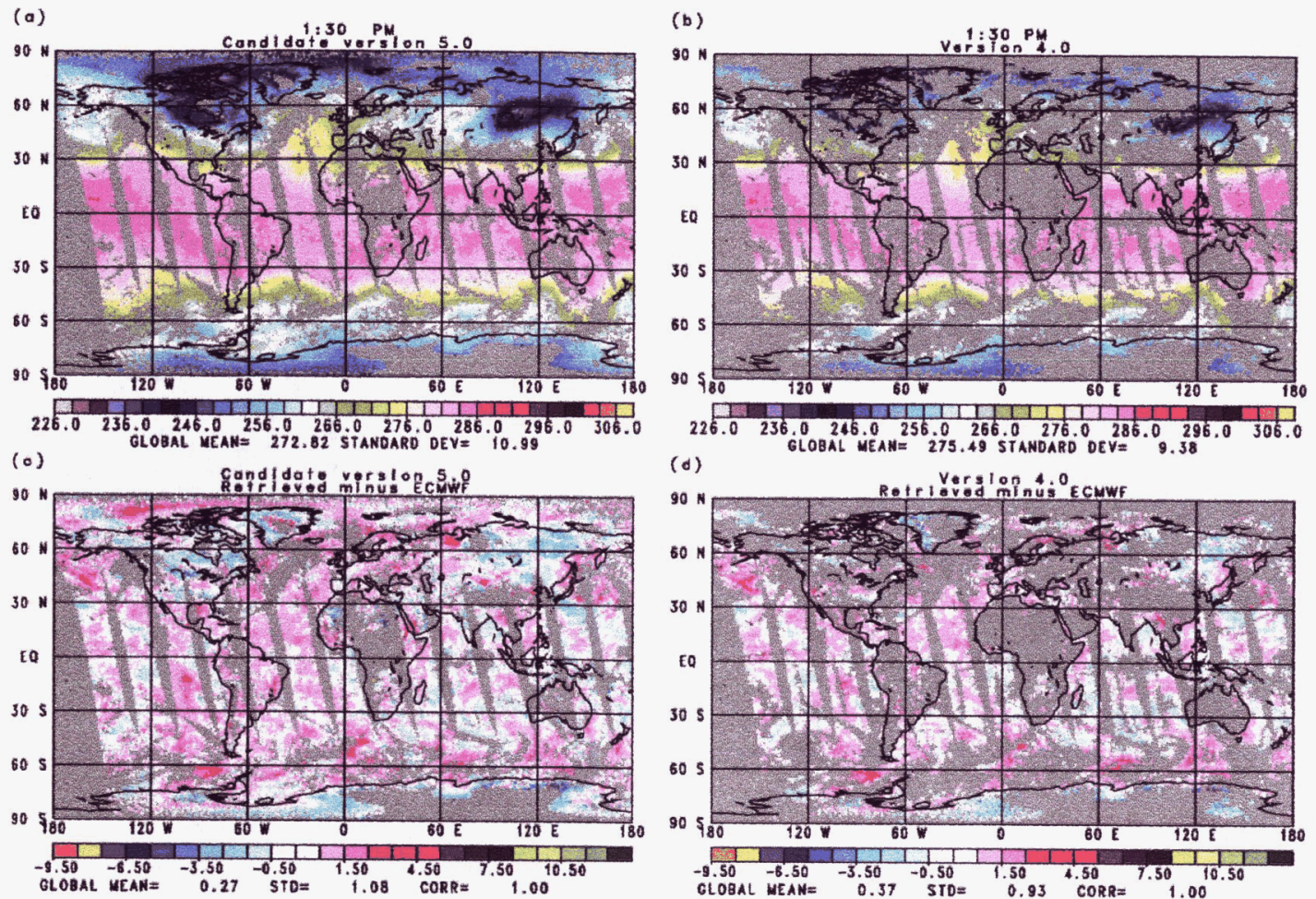

Figure 5

LAYER MEAN RMS TEMPERATURE ( ${ }^{\circ} \mathrm{C}$ ) GLOBAL DIFFERENCES FROY "TRUTH"

$$
\begin{aligned}
& \text { GLOBAL DIFFERENCES FROM } \\
& \text { JanUOYY } 25,2003 \\
& \text { GIOBO : }
\end{aligned}
$$
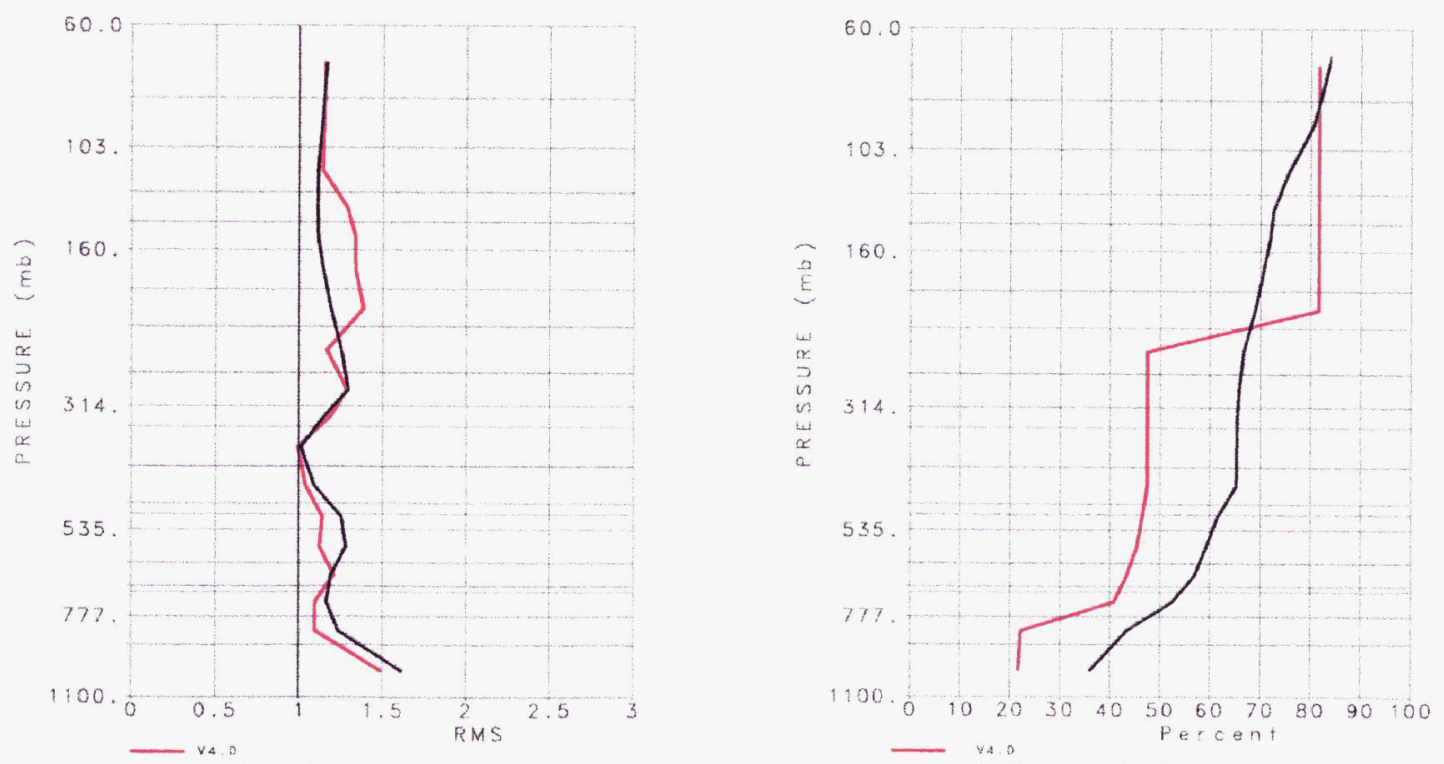

Figure 6 
Figure 6 shows RMS differences from ECMWF "truth" for global quality controlled Version 4.0 and candidate Version 5.0 retrievals, and the percent of all cases included in each set of statistics. The percent accepted at $70 \mathrm{mb}$ represents the percent of all cases in which successful IR/MW retrievals were produced. Candidate Version 5.0 has a much higher yield beneath $200 \mathrm{mb}$ than Version 4.0, with roughly comparable accuracy. Increasing spatial coverage of high quality retrievals is very important for both data assimilation purposes and climate and process studies.

\subsection{Error estimates and quality control for moisture profile}

The methodology used to generate error estimates for moisture profile is similar to that used for temperatures, but slightly different. Errors in the retrieved temperature profile and surface skin temperature will be major contributors to the errors in moisture profile. To reduce the dimension of the matrix $\mathrm{M}$, we express the moisture profile error estimate of $\delta \mathrm{q}_{\mathrm{i}}$ at all pressures as a linear combination of 6 temperature error estimates

$$
\delta \mathrm{q}_{\mathrm{i}}=\mathrm{M}_{\mathrm{ik}} \delta \mathrm{T}_{\mathrm{k}}(\mathrm{k}=1,6)
$$

where $\delta T_{k}$ corresponds to temperature error estimates at $150 \mathrm{mb}, 260 \mathrm{mb}, 500 \mathrm{mb}, 700 \mathrm{mb}, 850 \mathrm{mb}$, and $986 \mathrm{mb}$.

In the case of water vapor profile, the reported error $\delta q_{i}$ corresponds to the percentage error of the retrieved water vapor column density in layer i. $M_{\mathrm{ik}}$ is found which minimizes the layer i moisture weighted percentage error, $\mathrm{PE}_{\mathrm{i}}$ given by

$$
P E_{i}=\operatorname{RMS}\left[\left(q_{i}+q_{i}^{\text {truth }}\right)\left(\frac{q_{i}-q_{i}^{\text {truth }}}{q_{i}+q_{i}^{\text {truth }}}\right)\right] .
$$

In equation 3, the percent error is taken with respect to the average of the retrieved and true quantities to guard against very low "true" values and stabilize the problem. $\delta \mathrm{q}_{\mathrm{i}}$, determined using equation 2 , is to be interpreted as the percent error of the retrieved value of $q_{i}$ however.

The moisture profile error estimates are used only for error estimation purposes and not for quality control. Quality control for moisture profile is identical to that used for temperature profile, i.e., the moisture profile is classified as acceptable down to the same pressure level, $\mathrm{p}_{\text {good }}$, used to classify the temperature profile.

Figure 7a shows the percentage error of the retrieved water vapor in the lowest layer above the surface, which is roughly 250 meters thick, with regard to ECMWF truth, for all IR/MW retrievals. Figure $7 \mathrm{~b}$ shows the predicted fractional error, and Figure $7 \mathrm{~d}$ shows the difference between the predicted fractional error and the actual error. Agreement is quite good, with a spatial correlation of 0.63 . Figure $6 \mathrm{~b}$ shows quality controlled fractional errors compared to ECMWF for the surface, which includes only those cases for which $\mathrm{p}_{\text {good }}=\mathrm{p}_{\text {surf }}$.

Figures 8a shows RMS global percentage error of $1 \mathrm{~km}$ layer water vapor compared to ECMWF truth for quality controlled Version 4.0 and candidate Version 5.0 retrievals. As with candidate Version 5.0, Version 4.0 uses the same pressure dependent quality control for water vapor profiles as it does for temperature profiles. As with temperature profile, candidate Version 5.0 accuracy is comparable to that of Version 4.0, but with significantly greater spatial coverage. 

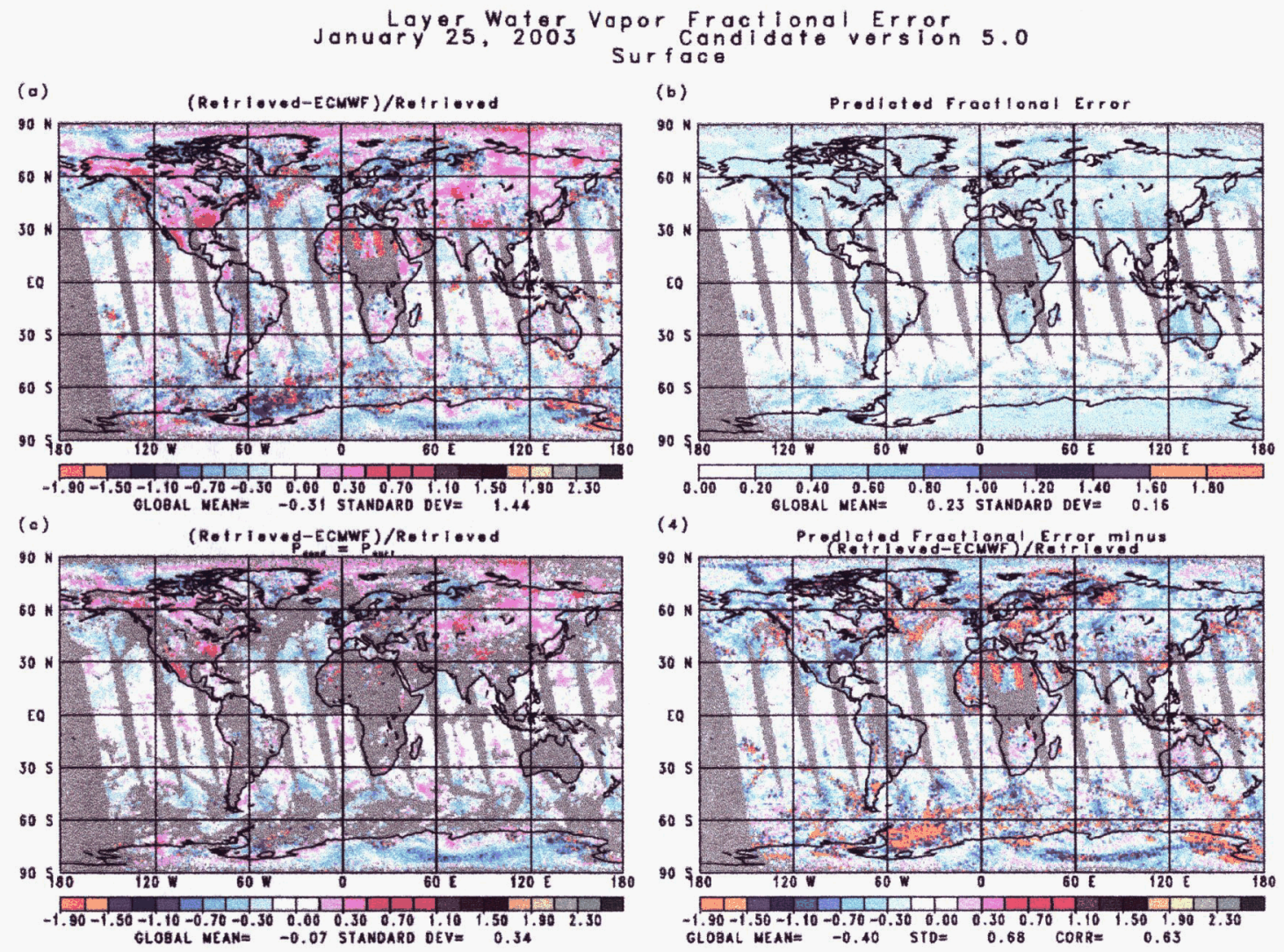

Figure 7
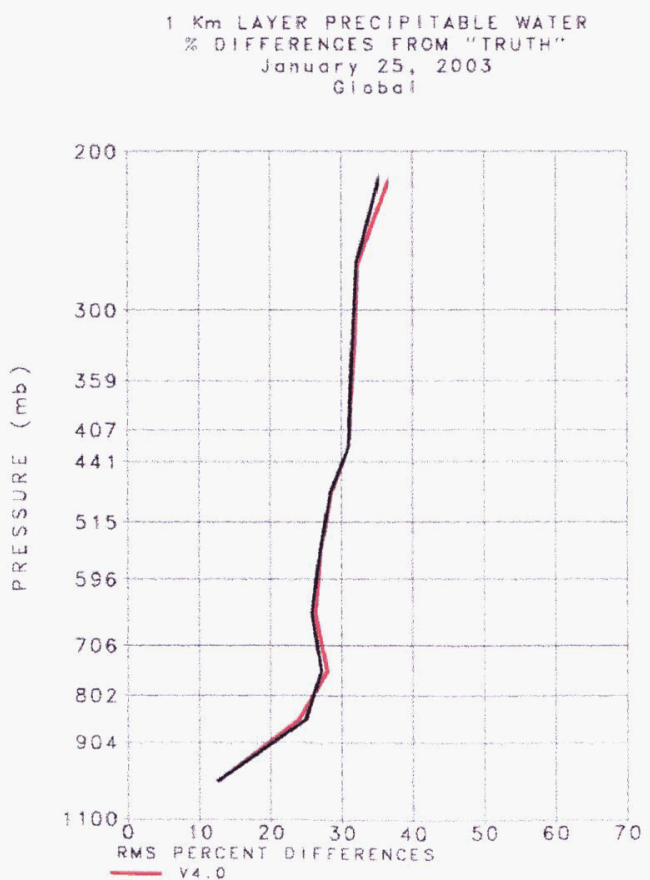
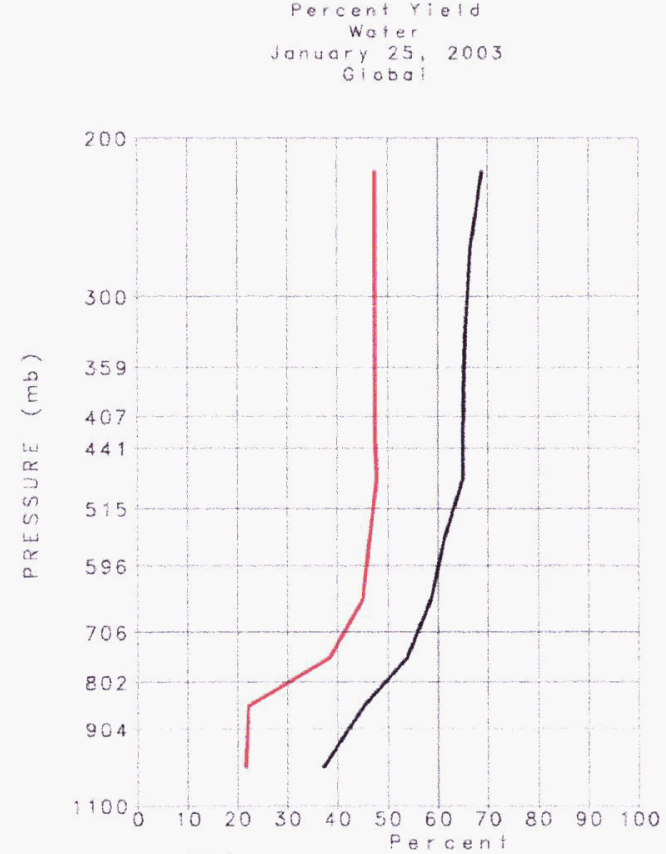

- va. Candidace va.0 Tiph Quatity control

Figure 8 


\subsection{Error estimates and quality control for clear column radiances}

Errors in cloud cleared radiances for channels $i, \hat{R}_{i}$, come from two sources. Even if the cloud clearing process were perfect, $\hat{R}_{i}$ would have errors due to noise in the observed radiance for channel $i$, with noise characteristics $N E \Delta N_{i}$. The cloud clearing process involves taking linear combinations of observed channel $\mathrm{i}$ radiances in 9 Fields of View and consequently introduces a case dependent noise amplification factor $A{ }^{2}$ Under these (perfect cloud clearing) conditions, the error in $\hat{R}_{i}$ would then be $\operatorname{AxNE} \triangle N_{i}$. The cloud clearing process is not perfect however and additional errors are introduced into $\hat{R}_{\mathrm{i}}$. These additional errors are the major cause of errors in the derived temperature profile. In.an analogous manner to what was done in generating the moisture profile error estimates, we model $\delta \hat{\mathrm{R}}_{\mathrm{i}}$ according to

$$
\delta \hat{R}_{\mathrm{i}}=\operatorname{AxNE} \Delta \mathrm{N}_{\mathrm{i}}+\sum_{1}^{6} \mathrm{M}_{\mathrm{ik}} \delta \mathrm{T}_{\mathrm{k}}
$$

and the $i^{\text {th }}$ column of the matrix $M$ (dimension $2386 \times 6$ ) is found by best matching RMS $\left(\hat{R}_{i}-\hat{R}_{i}^{\text {truth }}\right)$ where $\hat{R}_{i}^{\text {truth }}$ is the radiance computed using the ECMWF forecast as the truth.

Figure 9a shows a portion sample of an AIRS cloud cleared brightness temperature spectrum, $\hat{\Theta}_{i}$, where $\hat{\Theta}_{i}$ is given in brightness temperature units and is the temperature whose black body radiance is $\hat{R}_{i}$. The spectrum of clear column radiance error estimates for this case, in brightness temperature units, is shown in Figure $9 \mathrm{~b}$. The major spatial absorption features shown in Figure 9a are the $\mathrm{CO}_{2}$ absorption band, from $650 \mathrm{~cm}^{-1}$ to $750 \mathrm{~cm}^{-1}$ and the $\mathrm{O}_{3}$ absorption band from about $990 \mathrm{~cm}^{-1}$ to $1070 \mathrm{~cm}^{-1}$. Brightness temperatures correspond roughly to the temperature at the level of the absorption (or surface skin) at which most of the signal for that channel is coming from. The stronger the absorption in a given channel, the lower the brightness temperature for that channel. Some weak $\mathrm{CO}_{2}$ and $\mathrm{H}_{2} \mathrm{O}$ absorption lines (locally colder features) are also found in the "window" regions between $750 \mathrm{~cm}^{-1}$ and $990 \mathrm{~cm}^{-1}$, and $1070 \mathrm{~cm}^{-1}-1150$ $\mathrm{cm}^{-1}$, which are somewhat less sensitive to radiation coming from the surface than the surrounding channels. Errors in the cloud clearing process generally result in increasing clear column radiance errors as channels see deeper into the atmosphere, with the largest errors for those channels most sensitive to the surface. The clear column radiance error estimate spectrum shown in Figure $9 \mathrm{~b}$ is consistent with this expectation.

Figure 10a shows the spatial distribution of the differences of the derived clear column radiances for the AIRS channel at $724.52 \mathrm{~cm}^{-1}$ from those computed using ECMWF as truth for all cases in which a successful IR/MW retrieval was produced. This channel is between $\mathrm{CO}_{2}$ absorption lines, corresponding to a local maximum in the brightness temperature, and is primarily sensitive to atmospheric temperatures in the vicinity of $600 \mathrm{mb}$. There is a substantial negative bias due to insufficient cloud clearing in areas where poor retrievals were produced. Figure 10b shows the spatial distribution of the brightness temperature error estimates for this channel, and Figure 10d shows the difference between the error estimates and the absolute value of the "error". The spatial correlation is very good, with a value of 0.75 , and the magnitude of the predicted and observed errors match closely as well. Indeed, most of the largest differences occur at high latitudes, where the ECMWF "truth" may be of poorer quality. In these areas, low errors are generally predicted, and large "errors" are found. Most likely, these apparently under-predicted errors result from errors in the "truth", not in the clear column radiances. We use the predicted clear column radiance errors directly as quality control for the clear column radiances. Figure $10 \mathrm{c}$ shows the distribution of quality controlled clear column radiances, retaining only those cases in which $\delta \hat{R}_{\mathrm{i}}$ was less than or equal to $1.0 \mathrm{~K}$. Most of the negative bias has been eliminated. The standard deviation of the quality controlled clear column radiance errors is $0.81 \mathrm{~K}$, which is not significantly above the channel noise. 

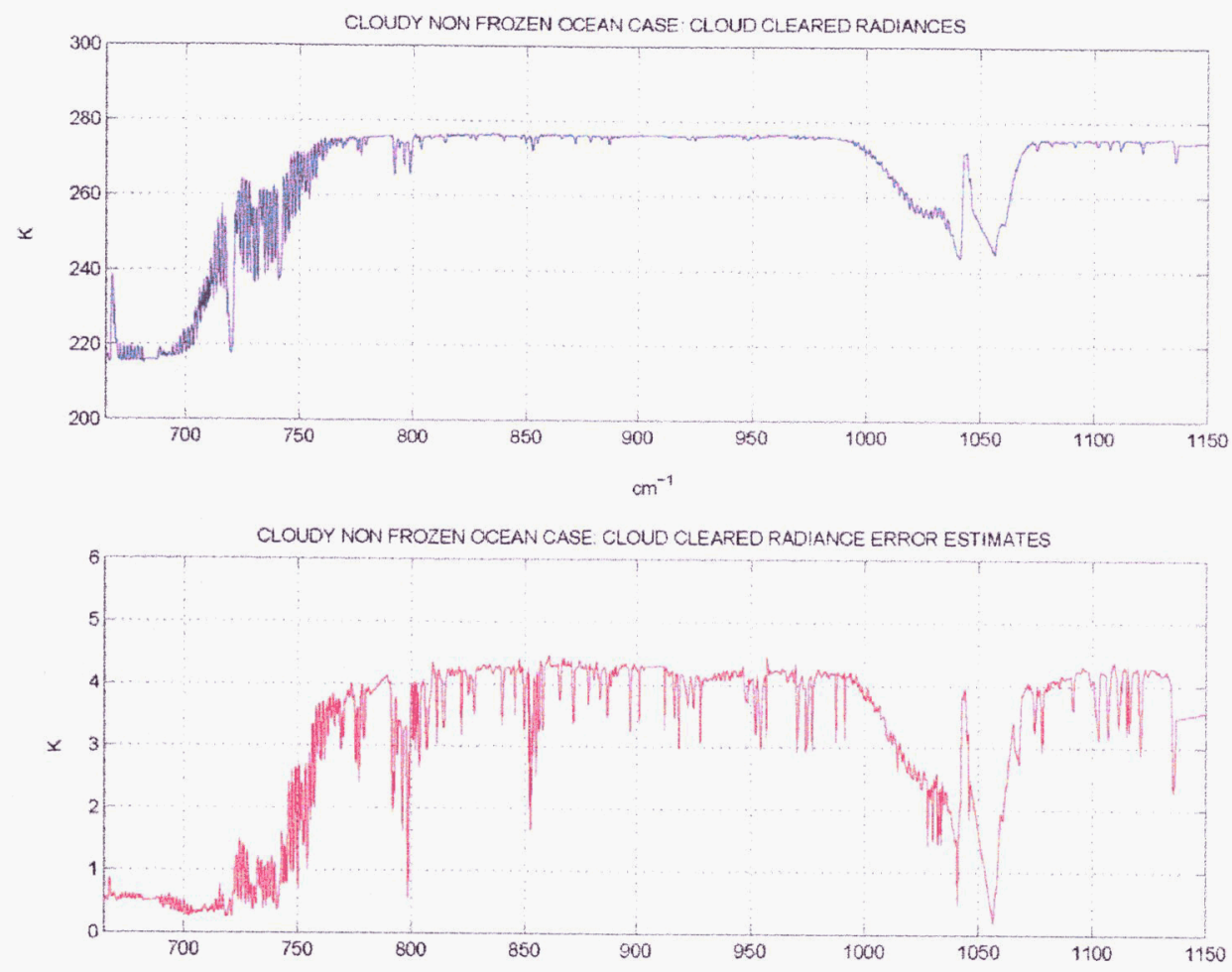

Figure 9

Cleor Column Brightness Temperature (K) January 25,2003
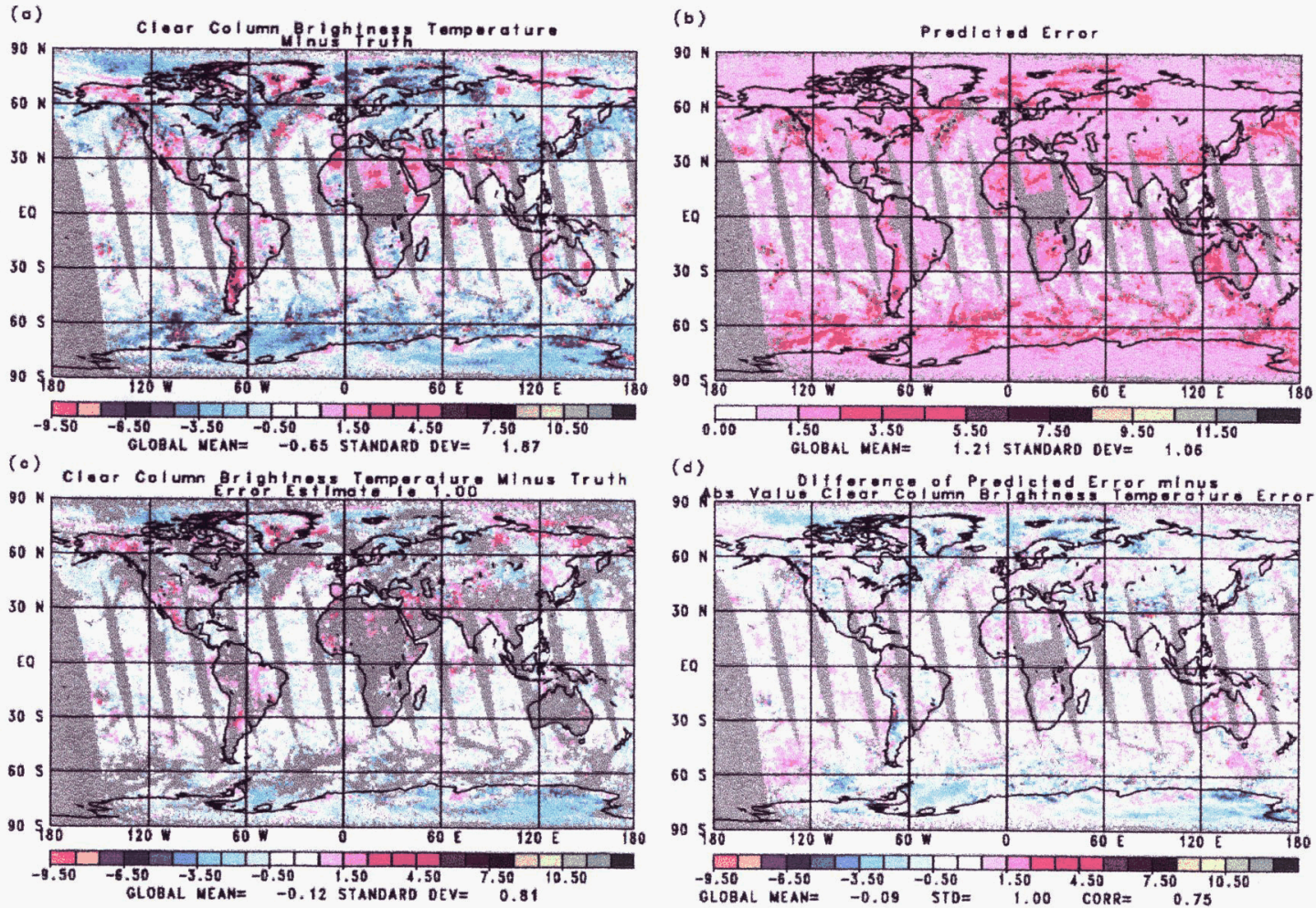

Figure 10 
Figure 11 shows a blow up of the global mean clear column brightness temperature spectrum and the quality controlled spatial standard deviation of the clear column brightness temperature errors obtained using candidate Version 5.0, which include only those cases in which $\delta \hat{R}_{\mathrm{i}}<1 \mathrm{~K}$, and obtained using Version 4.0. In Version 4.0, the mid-troposphere good criterion was used to accept clear column brightness temperatures and include them in the statistics. Also shown is the channel noise. The larger "errors" in clear column radiances are in fact a result of errors in the ECMWF "truth" . Version 5.0 quality controlled clear column brightness are highly accurate, and should produce good results when used for data assimilation purposes. The user can employ tighter acceptance criteria if they so desire, increasing accuracy at the expense of spatial coverage.

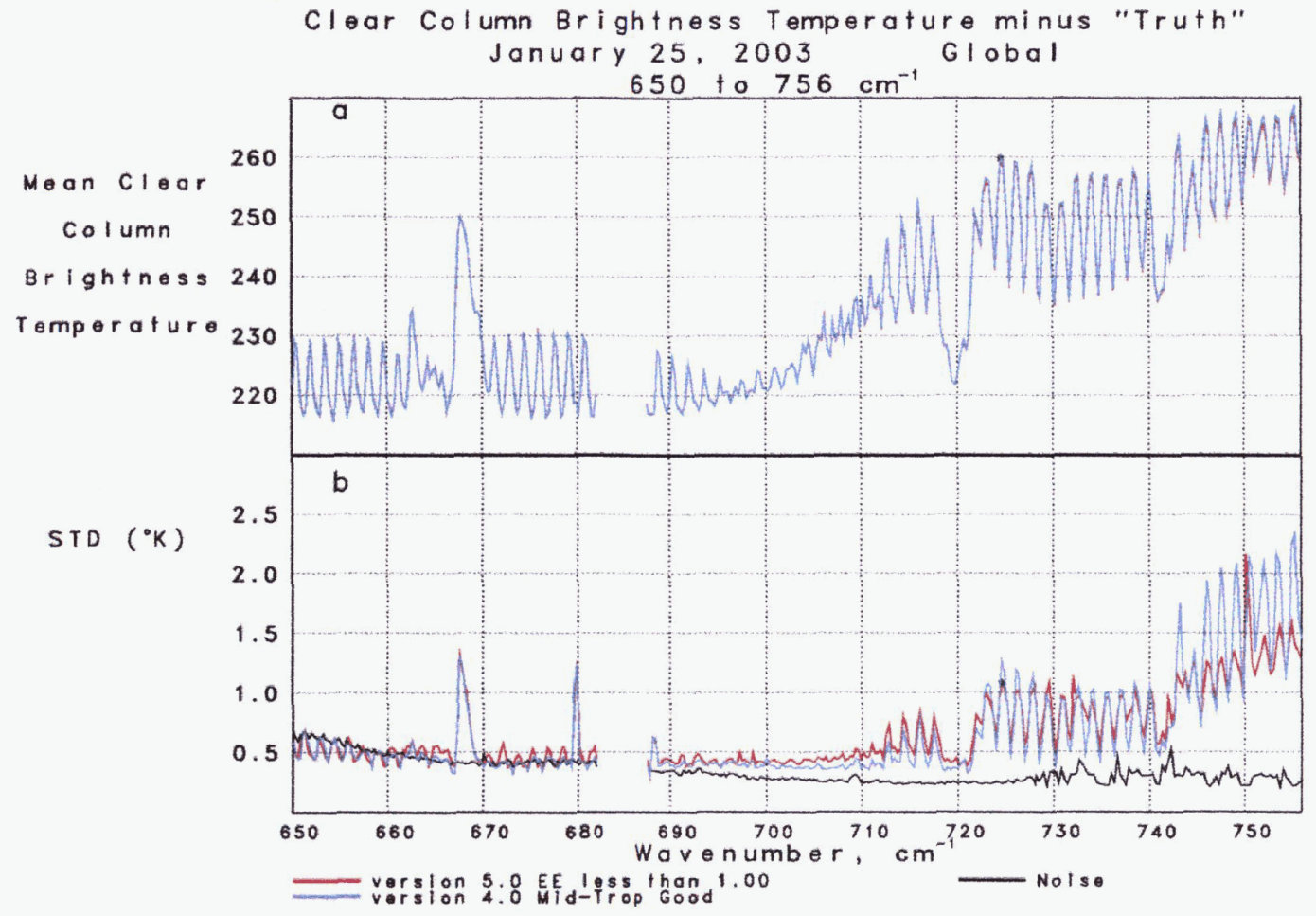

Figure 11

\section{REFERENCES}

1. Pagano, T. S., H. H. Aumann, D. E. Hagan, and K. Overoye, Prelaunch and in-flight radiometric calibration of the Atmospheric Infrared Sounder (AIRS). IEEE Trans. Geosci. Remote Sensing, 41, 265-273, February 2003.

2. Susskind, J., C. D. Barnet, and J. M. Blaisdell, Retrieval of atmospheric and surface parameters from AIRS/AMSU/HSB data in the presence of clouds. IEEE Trans. Geosci. Remote Sensing, 41, 390-409, February 2003.

3. Susskind, J., C. Barnet, J. Blaisdell, L. Iredell, F. Keita, L. Kouvaris, G. Molnar, and M. Chahine, Accuracy of geophysical parameters derived from AIRS/AMSU as a function of fractional cloud cover. J. Geophys. Res., 2006, in press.

4. Rosenkranz, P. W., Retrieval of temperature and moisture profiles from AMSU-A and AMSU-B measurements. In Proc. IGARSS, 2000.

5. Goldberg, M. D., Y. Qu, L. M. McMillin, W. Wolff, L. Zhou, and M. Divakarla, AIRS near-real-time products and algorithms in support of operational numerical weather prediction. IEEE Trans. Geosci. Remote Sensing, 41, 379-389, February 2003. 